\title{
ENTRE EL "PUBLICITY"Y LA GESTIÓN COMUNICATIVA INTEGRAL. EL CASO DEL FÚTBOL CLUB BARCELONA
}

\section{FROM PUBLICITY TO TOTAL COMMUNICATION MANAGEMENT. THE CASE OF THE FÚTBOL CLUB BARCELONA}

\author{
Fernando Olabe Sánchez \\ Universidad Miguel Hernández de Elche \\ molabe@umh.es
}

Resumen: El modelo comunicativo del FC Barcelona ha transitado desde la década de los 80 , cuando comienza a profesionalizarse la gestión de la información de los clubes de fútbol en España, hasta la actualidad, donde ha integrado la dimensión de la marca en la estrategia de difusión ante sus públicos objetivos. Para este trabajo se ha optado por una metodología cualitativa, con entrevistas en profundidad y una investigación empírica secundaria con fuentes bibliográficas y fuentes hemerográficas. Los resultados muestran que el club catalán ha pasado de la relación directa con los periodistas como canal para transmitir su imagen, a crear y gestionar sus informaciones a través de una multiplataforma de medios propios. Se concluye que el objetivo de este modelo es reducir su dependencia de las informaciones editadas por los medios de comunicación.

Palabras clave: comunicación corporativa, relaciones públicas, gestión de la comunicación, stakeholders
Abstract: The communication model of football club Barcelona has moved from the 1980s, when football clubs in Spain began to professionalize the management of information, until today when it has integrated the building of its brand with the dissemination strategy aimed at its target audiences. For this work, we opted for a qualitative methodology that included in-depth interviews and a secondary empirical examination of literature and newspaper sources. The results show that the Catalan club has gone from a direct relationship with journalists as a channel to transmit its image to using a multimedia platform of its own to create and manage its information. We conclude that the objective of this model is to reduce the club's reliance on information published by the media.

Key words: corporate communications, public relations, communication management, stakeholders. 


\section{Introducción}

El modelo de gestión del FC Barcelona parte de un principio inexorable que asumen sus dirigentes: los propietarios de los clubes son los socios, que eligen a sus presidentes en procesos electorales (Hamil et al., 2010), a diferencia de aquellos clubes de la liga española que se constituyeron en sociedades anónimas deportivas, en los que la propiedad la ostentan los accionistas mayoritarios. Este hecho, junto a su dimensión internacional, con millones de seguidores en todo el mundo ${ }^{1}$, provoca que la gestión empresarial y su visibilidad mediática se constituyan en la clave de su funcionamiento, donde, obviamente, también se incluye la trayectoria deportiva del club.

Pese a que, como resalta Fortunato (2010), los departamentos de comunicación de las organizaciones deportivas tienen como principal objetivo la atención a las relaciones con los medios de comunicación, la influencia de las entidades deportivas se extiende a otros colectivos con los que se relaciona y que también precisan de una gestión comunicativa específica, en función de sus características, perfiles y necesidades. En este sentido, Gómez y otros (2010) advierten que el mapa de públicos objetivos de los clubes de fútbol lo conforman los propietarios o socios, los aficionados de la entidad, los jugadores, el resto de clubes que participan en las competiciones (nacionales e internacionales), la federación, los patrocinadores y las instituciones públicas del país o zona donde está radicada la entidad deportiva. En el caso de los clubes españoles, los propios departamentos de comunicación de estas entidades reconocen que sus principales públicos objetivos son los abonados, los medios de comunicación y los patrocinadores (Sanahuja, 2013).

No obstante, conviene reseñar, como apuntan Chadwick y Arthur (2007), que en el desarrollo de los clubes de fútbol confluyen diversos factores e intereses, que determinan la transformación de sus estrategias comunicativas: los derechos televisivos, las estrategias de patrocinio de los clubes y de las instituciones deportivas (desde federaciones nacionales hasta organismos internacionales como la UEFA o la FIFA), el posicionamiento de marca, la aparición de nuevos soportes comunicativos y de marketing al amparo de las $\mathrm{TCl}$ y la propia globalización del deporte. Si en las últimas décadas del siglo XX la televisión contribuyó al lanzamiento del fútbol como un espectáculo de masas, trascendiendo su mera condición de disciplina deportiva, en el siglo XXI, con la irrupción de plataformas vinculadas a las $\mathrm{TCl}$, con el teléfono móvil, internet y la televisión digital y por satélite (Boyle y Haynes, 2004), el fútbol adquiere una dimensión empresarial, que se traduce en una cobertura informativa constante y global (Cleland, 2009).

Este escenario global acarrea importantes cambios para la actuación de los departamentos de Comunicación de los clubes de fútbol: por un lado, permite integrar las nuevas herramientas comunicativas que aportan las $\mathrm{TCl}$ y que posibilitan diferentes maneras de relacionarse con los públicos de estas entidades (Boyle y Haynes, 2009); por otro, como consecuencia de ello, se superan los modelos comunicativos tradicionales que empleaban estos departamentos, basados principalmente en las relaciones informativas con los medios tradicionales (Schultz et al., 2010). Los clubes han pasado a crear y gestionar sus informaciones a través de una multiplataforma

1 Al hecho de que en España sea uno de los clubes con los que más se identifican los españoles (según el Barómetro del CIS n².705, mayo de 2007), se añade que lidera el ranking de seguidores en redes sociales a nivel mundial, con más de 52 millones de fans en Facebook y más de 11 millones de seguidores en Twitter, según el estudio de la consultora Zenith Media. Recuperado de http://blogginzenith.zenithmedia.es/fc-barcelona-se-mantiene-como-el-club-deportivo-mas-popular-del-mundo-en-medios-sociales/ 
de medios propios, con el objetivo de reducir su dependencia de las informaciones editadas por los medios tradicionales (Moragas, et al., 2011).

\section{Metodología}

Para llevar a cabo este trabajo se ha optado por una metodología cualitativa. Se han empleado diferentes herramientas: a) entrevistas en profundidad a Toni Ruiz, jefe de prensa del FC Barcelona, que trabaja en el Club desde 1996 y b) una investigación empírica secundaria con fuentes bibliográficas y fuentes hemerográficas.

En primer lugar, para iniciar nuestra investigación y apoyándonos en la Grounded Theory (Glaser, 1992), se revisó la literatura académica de las principales revistas relacionadas con el management en el deporte, en las que se han buscado los ítems que nos interesan para a través de las siguientes palabras clave: comunicación corporativa en el fútbol, relaciones públicas en el fútbol, gestión comunicativa en el fútbol, Real Madrid CF, FC Barcelona y stakeholders en el fútbol.

Las revistas fueron seleccionadas de acuerdo con el listado de la International Media Management Academic Association (IMMAA). Otras publicaciones revisadas, que no se incluyen en ese listado, fueron seleccionadas por su especial relevancia con la gestión empresarial en el deporte. En cuanto a las fuentes hemerográficas, recurrimos a las hemerotecas online de los diarios El País, Abc, Mundo Deportivo y La Vanguardia, ya que su consulta en abierto agilizaba la investigación el acceso directo a documentos, en muchos casos en formato pdf.

\section{FC Barcelona, de las Relaciones Públicas (RR.PP.) con los medios a un modelo de gestión integral de la comunicación}

Con 115 años de historia, desde su fundación en 1899, el FC Barcelona se ha caracterizado por ser una entidad que ha sabido adaptarse a la propia evolución del fútbol como manifestación deportiva y a la dimensión empresarial y mediática que este deporte ha adquirido en las últimas décadas.

A lo largo de su historia, el FC Barcelona ha mantenido una estrecha relación con los medios, caracterizada por la interdependencia y por la necesidad de retroalimentarse mutuamente, aunque fuera en un escenario de amor-odio durante todos estos años (López, 1999). Estos vínculos se retrotraen a los mismos inicios del Club como entidad deportiva y a su fundador, el suizo Hans Gamper, que se sirvió de una nota de prensa, publicada el 22 de octubre de 1899, en la revista Los Deportes, para constituir lo que hoy se conoce como FC Barcelona (Bravo y Balmont, 2010).

Hasta que el Club se decidió a principios de los años 80 a constituir la figura del jefe de prensa, como observaremos a continuación, las relaciones entre el Club y los medios de comunicación se caracterizaron por el amateurismo, ya que no existía ningún profesional que las gestionara (Sanchís, 1999). Se daba la circunstancia de que durante años esa función la asumieron, en determinados momentos, los propios presidentes de la entidad, que redactaban las notas de prensa (Hernández, 1976).

No sería hasta 1980 cuando el Club instauró la figura del responsable de las relaciones con los medios (Serras, 2008), debido a la enorme expectación informativa que generaba la institución, tanto en la actividad deportiva de sus diferentes secciones, 
como en las cuestiones referidas a la gestión de la entidad. Los periodistas disponían de acceso directo a los protagonistas de la información, ya que entre ellos no existía ninguna barrera ni física ni institucional, lo que, en ocasiones, generaba conflictos por la ausencia de profesionales de la comunicación corporativa que gestionaran la relación entre las partes (Relaño, 2012).

Para analizar la evolución de la gestión de la comunicación del FC Barcelona, planteamos un recorrido con cuatro etapas: la primera, que se inicia en 1980, muestra los primeros pasos de la profesionalización de esta área en la entidad; la segunda focaliza la atención en la estrategia del Club, a partir de la década de los noventa, orientada a constituir sus propios soportes informativos para tratar de controlar los mensajes; la tercera, que comienza en 2003, con la llegada de Joan Laporta a la presidencia del Club, aborda cómo la entidad integró la comunicación como una herramienta estratégica en la gestión empresarial, y, por último, analizamos el modelo comunicativo del FC Barcelona con la implementación de las tecnologías de la información y la comunicación en la gestión integral.

\subsection{Profesionalización de la comunicación: década de los ochenta}

EI FC Barcelona fue el primer club español en incorporar un profesional de la comunicación en su estructura organizativa, con el objetivo de gestionar las relaciones que la entidad mantenía con los periodistas que cubrían la actualidad deportiva e institucional (Perearnau, 2003). El proceso de profesionalización de la comunicación del Club se inició durante la presidencia de José Luis Núñez (1978-2000), cuando éste incorporó en octubre de 1981 al periodista del diario Mundo Deportivo Ricard Maxenchs como jefe de prensa, quien permaneció en el cargo durante 20 años. Maxenchs fue reconocido por la mayoría de los periodistas que cubrían la actualidad informativa del FC Barcelona como el paradigma de lo que debe ser un director de comunicación en un club de fútbol, ya que logró canalizar de forma eficaz y sin estridencias las relaciones entre los periodistas deportivos y el Club (Serras, 2008).

No obstante, su labor no fue sencilla. En sus primeros años de mandato, la preocupación de Núñez pasaba por recuperar la economía del Club, que se encontraba en números rojos (García Candau, 1979). Esa gestión, sin embargo, se caracterizó por los constantes enfrentamientos con los medios y con los periodistas deportivos, llegando a vetar el acceso al Camp Nou del redactor-jefe de Deportes de El Periódico de Catalunya por publicar determinadas informaciones que cuestionaban el comportamiento del Club (Bernardos, 1979)2. Maxenchs tuvo que desarrollar su trabajo en ese clima de tensión, con un presidente del que criticaba la excesiva atención mediática sobre el Club y que llegó a reclamar la desaparición de la mitad de los rotativos editados en Barcelona (García Candau, 1980).

El estilo de Maxenchs se caracterizó por conocer las necesidades de los medios y satisfacerlas en la medida de lo posible, con elegancia, mano izquierda y conocimiento profundo de las rutinas productivas de los periodistas (Escorcia, 2010), ya que antes de poner en marcha el departamento de Comunicación del FC Barcelona había trabajado en radio, prensa y televisión. En el Club se implantó, progresivamente, una dinámica profesional en las relaciones de los jugadores con los medios. Si hasta esa época predominaba la facilidad con la que los periodistas conseguían las declaraciones de los futbolistas de forma directa y sin intermediarios, Maxen-

2 El periodista Alex Botines publicó un reportaje en el que informaba sobre las irregularidades del expediente de traslado militar del jugador Carrasco. El club no llegó a desmentir el contenido del reportaje y se limitó a declarar persona non-grata al periodista (Bernardos, 1979). 
chs estableció la normativa de que los jugadores advirtieran, previamente, al jefe de prensa, su intención de atender la solicitud de entrevista de los medios. Asimismo, la capacidad de gestión de Maxenchs impulsó una estrategia de visibilidad del Club fuera de Cataluña, que propició la constitución de más de 1.500 peñas de seguidores barcelonistas en toda España (Perearnau, 2003).

La atención mediática se incrementó a mediados de esa década y a principios de los noventa, cuando surgieron nuevas empresas periodísticas, desde las ediciones catalanas de grupos periodísticos madrileños, como Recoletos, Prisa, Prensa Española y Unedisa (Jones, 2004). Asimismo, también se incrementó el interés por la información deportiva, tanto por parte de los diarios especializados y generalistas como de las revistas y las cadenas de radio (nacionales y autonómicas), que incorporaron espacios específicos (Jones, 1994). Por lo que respecta a la televisión, la incorporación de las cadenas privadas y autonómicas provocó que la información deportiva adquiriera mayor visibilidad, hasta el punto de que en 1993 el 10\% de la programación televisiva en España estaba dedicada al deporte, en sus diferentes formatos: retransmisiones de eventos, programas de actualidad deportiva e información en los noticiarios (García et al., 1995).

La gestión comunicativa del Club se dividió en dos ámbitos: la información deportiva y la institucional, aunque siempre desde una perspectiva de relaciones informativas con los medios de comunicación como públicos objetivos. La información deportiva recaía en el gabinete de prensa y se caracterizó por su capacidad de relacionarse con los medios atendiendo a sus necesidades y sin privilegiar a unos respecto de otros, teniendo en cuenta la idiosincrasia de los periodistas deportivos (Escorcia, 2010). Asimismo, desde este departamento también se gestionaban las acciones de Relaciones Públicas del Club y el protocolo, tanto en eventos sociales como deportivos. Para todo ello, el departamento contaba con una estructura compuesta por cinco personas, como asegura Ruiz ${ }^{3}$.

El modelo comunicativo del Club en aquella época se configuró, por tanto, en una cierta contradicción: por un lado se buscaba la reconciliación con los periodistas deportivos, pero, por otro, se seguía privilegiando a determinadas cabeceras. Mientras Maxenchs lidiaba con el día a día de la gestión comunicativa en la esfera deportiva, Gené y los siguientes asesores del presidente, como Pere Oriol Costa o Domènec García, gestionaban la comunicación institucional del Club, con una política de filtraciones interesadas a determinados medios (Santos, 2010).

\subsection{El control de la información: la creación de los medios propios de informa- ción en la década de los noventa}

La presidencia de Núñez estuvo marcada por los desencuentros con los medios de comunicación, y por el control a los periodistas deportivos a través del director de comunicación de la entidad, Domènec García (Mundo Deportivo, 2000). Precisamente, el papel de Domènec García fue determinante en el comportamiento comunicativo del Club.

En esos años, García adquirió mayor peso específico a la hora de establecer la política comunicativa de la entidad y generó diversos enfrentamientos con los medios, que lo acusaron de gestionar discrecionalmente las acreditaciones de los periodistas, vetar el acceso de algunos de ellos a las instalaciones del Club e incrementar la política de filtraciones (Santos, 2010).

3 Entrevista personal mantenida con el jefe de prensa del FC Barcelona. 
Ante esa situación, el Club puso en marcha sus propios canales de comunicación, cuyo objetivo pretendía crear la propia agenda temática dirigida a socios y seguidores de la entidad, que los medios tradicionales no contemplaban. Esta decisión confirió al Club una actitud activa en el ecosistema informativo sobre la actualidad del FC Barcelona, a diferencia de épocas anteriores, en las que el Club se comportaba de forma pasiva, a disposición de lo que marcaban los medios de comunicación, que destinaban una amplia cobertura a la actualidad del Club y que llegaban a marcar la agenda temática de la entidad (Santos, 2010: 35-38).

En este sentido, el Club se ha caracterizado, desde la presidencia de Núñez, por apoyarse en determinados periódicos catalanes, con los que ha establecido una relación que, según Ginesta y otros (2011), puede ser calificada de "parasitaria", dada la dependencia económica que esos medios tienen del Club. La amplia cobertura mediática en los periódicos deportivos catalanes (Sport y Mundo Deportivo) muestra el grado de dependencia mutua que mantienen el Club y los medios de comunicación, hasta el punto de que estos medios publican más información generada por el Club que la producida directamente (Xifra, 2008).

Para el ex director de Comunicación del Club, Jordi Badía (2009), el FC Barcelona estableció, desde hace años, una doble política de relaciones con los medios: por un lado, la puramente informativa, en la que el departamento de Comunicación gestiona los productos y contenidos noticiosos; y por otra, la comercial, en la que el Club se apoya en los medios de comunicación, concretamente en los catalanes, para lanzar a sus seguidores diversos productos de merchandising, a la par que los medios utilizan esas promociones para incrementar su ventas.

En este escenario, durante la presidencia de Núñez, el FC Barcelona inicia el proceso de gestionar la información que generaba el Club sin la necesidad de la intermediación de los medios tradicionales. A este respecto, el Club lanzó sus propios soportes comunicativos, como el canal de televisión o los boletines informativos para los socios. Los soportes comunicativos que puso en marcha el Club dependían del área de Presidencia del Club y estaban coordinados por Domènec García (Miserachs y Polo, 1999). Entre ellos, figuran los siguientes:

- Canal FC Barcelona. Fue el antecedente del actual FC Barcelona TV y se emitía a través de Vía Digital. Las emisiones se iniciaron el 27 de julio de 1999 y en la programación de ese día se emitió el partido que el FC Barcelona ganó al Real Madrid en el Bernabéu por 0-5 cuando fichó a Cruyff como jugador. La parrilla estaba diseñada con retrasmisiones de los entrenamientos y partidos de las distintas competiciones, espacios para entrevistas a directivos, técnicos y jugadores, con dos informativos diarios, a las 14.00 y a las 20.00 (Rodríguez de Paz, 1999).

- La Veu del Club. Aunque se constituyó en 1998 como un boletín destinado a informar a los socios, esta revista era deudora de los diferentes modelos de publicaciones internas que existían desde 1921. El antecedente más cercano, también durante la presidencia de Núñez, fue Fem FC Barcelona: La revista del FC Barcelona. La Veu del Club pasó por diferentes etapas hasta convertirse en la actual FC Barcelona.

- Sitio web www.fcbbarcelona.cat. El Club presentó su primer sitio web corporativo el 12 de junio de 1997 (Mundo deportivo, 1997). En sus inicios se trataba de un soporte puramente informativo, que fue incrementando sus prestaciones y servicios a medida que avanzaba el desarrollo de la propia estructura universal de la red. 
La llegada de Joan Gaspart a la presidencia en julio de 2000, durante el periodo intermedio entre Núñez y Laporta, promovió la primera reorganización del área de Comunicación del Club, que se constituyó en una de las apuestas decididas del nuevo presidente para reestructurar la entidad (Allen y Eguía, 2005). Toni Ruiz, que se incorporó al departamento de comunicación en 1996, fue nombrado jefe de prensa del FC Barcelona en agosto de 2001, en sustitución de Ricard Maxenchs, quien pasó a desempeñar funciones ejecutivas en el Área Social e Institucional de la entidad, como director general adjunto (Mundo Deportivo, 2001).

\subsection{La comunicación integrada en la gestión empresarial: 2003-2010}

Con Joan Laporta como presidente (2003-2010) se modifica el modelo de gestión, completamente diferente del promovido por Josep Lluis Núñez, que durante 22 años se había caracterizado no sólo por un estilo empresarial agresivo, en el que se buscaba maximizar el potencial económico del Club, sino por la pérdida de transparencia dentro de la institución (Hamil et al., 2010). Para ello, el equipo directivo de Laporta definió cuatro líneas básicas: a) priorizar los éxitos deportivos, b) la transparencia en la gestión, c) implementar estrategias financieras que dotaran de recursos propios al Club y d) posicionar al FC Barcelona, a través de diferentes acciones de Responsabilidad Social Corporativa (RSC), en un referente social de ámbito internacional.

Estos planteamientos se fundamentaban en la convicción de que el fútbol debía contemplarse como un negocio. Por ello, el FC Barcelona podía desenvolverse como una entidad de ámbito global, a diferencia de otros clubes menores, y ese negocio debía orientarse a la industria del entretenimiento, al modo de otras compañías internacionales como Walt Disney (Dávila et al., 2007).

Laporta y su junta directiva iniciaron un proceso acelerado para recuperar la transparencia en la gestión de la entidad, aplicando buenas prácticas de gobernanza e impulsando iniciativas de RSC que reforzaran la reputación (Hamil et al., 2010). La RSC era la consecuencia natural de aplicar el modelo de gestión del "Círculo Virtuoso", en el que estaban implicadas tres áreas: deportiva, económica y social. El objetivo de estas acciones era claro: reforzar la imagen de marca, que ya se había diferenciado con el lema "Mès que un Club" (Dávila et al., 2007; Xifra, 2008), e incrementar sus valores corporativos como una entidad cosmopolita y abierta (Portabella, 2010), aunque la inversión en acciones sociales podría afectar a su crecimiento comercial (Chadwick y Arthur, 2007).

Como advierten Chadwick y Arthur (2007), uno de los aspectos que caracteriza al FC Barcelona respecto de otros clubes es su capacidad para desarrollar estrategias innovadoras. Con Joan Laporta llegó la profesionalización al Club, en todas las áreas estratégicas que le permitieran gestionar de forma efectiva los valores y atributos del Club desde una perspectiva innovadora en cuanto a los modelos de gestión de sus predecesores en el cargo (Allen y Eguía, 2005). El objetivo era impulsar al FC Barcelona más allá de su famoso eslogan "Més que un Club" y posicionarlo como un referente mundial del deporte (Hamil et al., 2010), para lo que se apostó por las Tecnologías de la Información y la Comunicación (TIC), especialmente Internet, como un canal de comunicación entre la entidad y sus públicos objetivos (Estanyol, 2010).

La estrategia para relacionarse con sus stakeholders se fundamentó en diversas actuaciones sobre las áreas claves a la hora de gestionar la imagen del Club. La comunicación se diseñó de forma holística, con una planificación transversal que integraba a las áreas de Marketing, Relaciones Institucionales, Protocolo y Medios (Ordeix y Ginesta, 2009), que permitiera incrementar los canales de comunicación propios, especialmente con los socios y con los seguidores (Hamil et al. 2010). 
En julio de 2003, Laporta designó como director de comunicación a Jordi Badía, jefe de deportes del diario catalanista Avui, periódico que le apoyó con mayor decisión que el resto de cabeceras catalanas durante las elecciones a la presidencia de la entidad (Santos, 2010). El director de comunicación asumió funciones directivas durante sus cinco años en la entidad, al mismo nivel que otros miembros de la junta de Laporta, aunque al principio se integró, orgánicamente, en el área estratégica de Marketing (Allen y Eguía, 2005).

Badía estructuró el departamento a partir de una premisa: recuperar la gestión total de los medios propios del Club, que en muchos casos estaban externalizados (Badía, 2011). Al mismo tiempo, lo dotó de mayor responsabilidad y contenido, lo que sentó las bases para que los socios y aficionados del Club obtuvieran la información generada por la entidad a través de los propios soportes del Club y no solamente con la intermediación de los medios tradicionales (Badía, 2009). El área de Comunicación asumió competencias en la gestión de contenidos del sitio web corporativo, con la incorporación de Andrea Balducci, hasta esas fechas responsable del sitio web del Inter de Milán. También coordinó las relaciones con los medios de comunicación de todas las secciones del Club y los medios propios del mismo, como el sitio web corporativo, la revista FC Barcelona y el canal FC Barcelona TV. A ello se añadía el gabinete de prensa, con Toni Ruiz como responsable (Segura, 2004).

Durante su gestión al frente de la comunicación del Club, se determinó el papel de este departamento en muchos aspectos que incidían en la imagen que la entidad quería trasmitir a la opinión pública y en las relaciones con los medios de comunicación. Según Badía (2009), hasta la llegada de Joan Laporta a la presidencia del Club, la gestión comunicativa de la entidad se basaba únicamente en la relación con los medios de comunicación, con el añadido de que en muchos casos, directivos y presidentes mantenían, además, su propia dinámica de relaciones personales con los periodistas más allá de las pautas del departamento de comunicación, con filtraciones y escasa unidad y coherencia en los mensajes institucionales.

La gestión comunicativa del FC Barcelona estaba planteada desde una perspectiva colaboradora con los medios, aunque el control de la información que generaba el Club lo realizaba directamente el propio departamento de comunicación, quien supervisaba la relación de jugadores y directivos con los periodistas. Esta normativa generó diversos encontronazos con miembros de la Junta Directiva, que se saltaban estas normas o las criticaban (Badía, 2009). Ello provocó, asimismo, críticas de los periodistas deportivos, que entendían estas medidas como una política de aislamiento del Club hacia los medios tradicionales (Santos, 2010).

Asimismo, el departamento de Comunicación implantó una nueva estrategia con los medios. Si bajo el mandato de Núñez la relación con los medios catalanes se entendía desde la perspectiva del enfrentamiento, lo que conllevó un aislamiento político del Club (Hamil et al., 2010), con la gestión de Laporta, los medios se convirtieron en rehenes de la información que gestionaba la entidad a través de sus propios soportes y con una redacción propia integrada en el departamento de comunicación (Ordeix y Ginesta, 2009). Ello degeneró en un conflicto con los medios que habitualmente cubrían la actualidad del Club y que lo consideraban como un atentado contra la libertad de expresión (Santos, 2010).

La estrategia del FC Barcelona se fundamentó en la relevancia que adquirieron los medios propios como altavoces institucionales, a través de la revista corporativa, los newsletter, o el canal propio de televisión del Club, FC Barcelona TV, entre otros (Ginesta, 2010). Asimismo, se definió una visión de proyección internacional, ya que 
a través de las emisiones televisivas en FC Barcelona TV (con contenidos de la Liga de Campeones, con resúmenes de los partidos de la competición española, con espacios sobre con la actualidad de otros equipos de la entidad y con reportajes especializados) se llegaron a acuerdos para su distribución en más de 50 países. A ello se unió el desarrollo tecnológico del sitio web corporativo del Club, con emisiones en streaming y con la implementación de diversas aplicaciones para móviles dirigidas a sus seguidores en todo el mundo (Dávila et al., 2007).

En este escenario, el FC Barcelona implementó un mix de la comunicación, en el que se integran los soportes online y offline para gestionar las relaciones con todos los públicos objetivos de la entidad, entre los que se incluyen a los socios, los aficionados y las peñas barcelonistas (Ginesta, 2008). La gestión de estos soportes dependía de la dirección de comunicación del Club. El departamento de comunicación estaba configurado por 38 personas en plantilla y 48 subcontratadas a la productora Mediapro, aunque en la gestión de los contenidos colaboraba el área de Marketing (Ginesta, 2009). La estructura mediática del Club catalán desde principios del siglo XXI está configurada por los siguientes soportes, que coinciden mayoritariamente con los que pusieron en marcha las anteriores juntas directivas de Núñez y Gaspart, aunque enfocados a un concepto más mercantil:

- FC Barcelona TV. Recuperada la gestión de los contenidos que estaban en manos de Mediapro (aunque en esta etapa se mantuvo como partner tecnológico), se procedió al cambio de denominación del anterior Canal FC Barcelona. Su emisión pasó por diversas plataformas: Digital +, Imagenio de Telefónica, ONO y en abierto por TDT. Con un 95\% de producción propia, que el canal distribuía también internacionalmente en inglés, su programación estaba diseñada con contenidos informativos sobre la actualidad del Club, a través de noticiarios y reportajes, y con la retrasmisión en directo de ruedas de prensa, entrenamientos y partidos del equipo de fútbol.

- FC Barcelona TV online. Canal de suscripción en zona Premium de la web del Club, que se emitía en catalán, castellano e inglés. La programación se diseñó para poder descargarse, entre otras opciones, vídeos de los fichajes del Club, programas de FC Barcelona TV, partidos históricos o vídeos de la actualidad de las diferentes secciones de la entidad.

- Canal FC Barcelona TV en Youtube. Es la primera aproximación del Club a los Social Media. El canal de Youtube se lanzó en 1997 en España y se amplió al mercado internacional para Japón, China y Oriente Próximo.

- Sitio web corporativo. Se constituyó en el eje vertebrador de la comunicación del Club con sus diferentes públicos, ya que se configuró como la plataforma en la que integrar los diferentes medios de comunicación de la entidad. Asimismo, se amplió la estrategia comercial, de marketing y relacional con los seguidores de todo el mundo en seis idiomas: catalán, castellano, inglés, japonés, chino, árabe y coreano. Desde el departamento de comunicación se pretendía, además, que fuera una herramienta de trabajo a la que pudieran acudir los periodistas deportivos, con la publicación de noticias propias sobre la actualidad del Club.

- R@dio FC Barcelona. Integrada en la web del Club, desde donde se emite. R@ dio FC Barcelona se inauguró en 2007 y su parrilla se ceñía inicialmente a la retrasmisión de los partidos de los equipos de fútbol, en castellano, catalán e inglés, y de baloncesto, en este caso únicamente los de la Liga Europea. 
- Servicios móviles. Herramienta de marketing y de comunicación, diseñada para que los seguidores y socios del Club se pudieran descargar logotipos, melodías, tonos, fondos de pantalla y video clips, así como recibir alertas SMS y MMS, con informaciones de servicio sobre la entidad. En 2005 generó unos ingresos de casi 1,8 millones de euros.

- Revista FC Barcelona. Publicación bimensual, que se distribuye entre los socios del Club, cuyos contenidos se editan en catalán y castellano. En la temporada 2007-2008, el formato de informaciones variadas pasó a monográficos sobre un aspecto concreto de la entidad, en función de la actualidad. Esa temporada empezó a encartarse el boletín FC Barcelona Junior, suplemento dirigido a los socios más jóvenes y a las familias.

- Periódico Camp Nou. Publicación que se edita los días que el primer equipo de fútbol juega su partido en el estadio barcelonista. Se edita en catalán, la lengua oficial del Club, y los contenidos combinan información sobre el partido y reportajes.

- FC Business. Publicación dirigida a los patrocinadores y socios comerciales de la entidad. Se edita en catalán, castellano e inglés, y ofrece información exclusiva para este colectivo, desde estadísticas a reportajes sobre la activación de los patrocinios.

El modelo del FC Barcelona se considera uno de los que mejor desarrollan la difusión de noticias especializadas y la producción de profesional de contenidos, tanto audiovisuales como textuales, ya sea gestionándolos el propio Club o externalizando algunos de los servicios (Hutchins et al., 2010). No hay que olvidar que el FC Barcelona ha tenido un papel pionero en el uso de las TIC como estrategia comunicativa, lo que le ha ayudado a manejar todos sus mensajes institucionales con sus diferentes públicos objetivos en el marco de club global en el que actúa (Ginesta y Casino, 2008).

\subsection{El modelo comunicativo del FC Barcelona en la actualidad}

Desde la entidad azulgrana se entiende que la puesta en marcha de este modelo comunicativo está diseñada para responder ante un público que no se ciñe únicamente a los medios ni los periodistas deportivos: su objetivo es lograr una voz propia ante los socios y los millones de seguidores en todo el mundo, que precisan de una información institucional que no esté condicionada por la actual sobredimensión de medios, que cubren constantemente al FC Barcelona y que provocan un ruido comunicativo que el Club quiere controlar.

Partiendo de esta premisa y a través de los datos de las memorias anuales del Club y de la información suministrada por el adjunto al director de Comunicación, hemos elaborado el mapa de la gestión comunicativa que realiza el Club, diseñada para atender a tres perfiles comunicativos diferentes: a) las relaciones con los medios de comunicación y los periodistas deportivos, b) la comunicación con los socios y aficionados y c) la comunicación de marketing.

El modelo actual del FC Barcelona se fundamenta en dos premisas: por un lado, las relaciones habituales con los periodistas deportivos, que mantienen su función de lograr publicity sobre la entidad (Favorito, 2007), basada, fundamentalmente, en herramientas y acciones off line como las ruedas de prensa, los comunicados de prensa, la gestión de entrevistas (personales o grupales) antes y después de los partidos y la comunicación personal (Pedersen et al., 2007); por otro lado, la gestión de contenidos a través de los medios vinculados a las TIC, que favorecen el acceso directo 
a los públicos y la globalización de la entidad (Boyle et al., 2004), y que permite a través de las multiplataformas de los New Media ${ }^{4}$ desarrollar estrategias específicas vinculadas a la gestión empresarial (Santomier et al., 2010).

\subsubsection{Relaciones con los medios de comunicación}

El funcionamiento del departamento de Comunicación del FC Barcelona se desarrolla en tres ámbitos, condicionado por la constante atención mediática que recibe la entidad, tanto en España como en la esfera internacional:

- Dar respuesta a las peticiones de los medios. Muchas veces, el departamento de Comunicación actúa como filtro, sobre todo cuando se refiere al primer equipo de fútbol, ya que tiene que atender un elevado número de peticiones de entrevistas y reportajes de diferentes medios. "La petición es tan extraordinaria, que nuestro activo no es suficiente para dar respuesta a todas esas peticiones, con lo cual el departamento de Comunicación actúa como filtro", asegura Toni Ruiz ${ }^{5}$. Según la memoria anual del Club, en la temporada 2012-2013 se acreditaron más de 10.000 profesionales entre periodistas, técnicos de sonido, cámaras, fotógrafos, productores y comentaristas.

- Como departamento de comunicación al uso. Cuando se ha de transmitir información que no es estrictamente deportiva, sino relativa a la gestión de las otras secciones del Club, de la fundación o de la propia institución. El departamento de comunicación no solo atiende a los periodistas que siguen la actualidad del primer equipo de fútbol, sino que también ha de gestionar la información que genera la actividad de las secciones de baloncesto, balonmano, hockey y fútbol sala, la del fútbol formativo, a la prensa internacional que se preocupa por el fenómeno y los proyectos generados por el departamento comercial y la fundación.

- La redacción de contenidos. El departamento de comunicación ha diseñado una estrategia para dar cobertura a las necesidades de los medios y dotar de contenidos exclusivos a sus públicos estratégicos. "El departamento de Comunicación tiene una redacción propia que prepara contenidos diariamente para distribuir a nuestros socios, a nuestros aficionados y también a los medios, porque actuamos de agencia, a través de diferentes medios de comunicación", asevera Ruiz.

Desde el Club se entiende que el diseño de este comportamiento comunicativo responde a una política de transparencia con sus socios. A través de las encuestas que realizan con los socios de la entidad, el departamento de comunicación ha advertido que estos compatibilizan la información sobre el Club de los canales propios con la que emiten los medios de comunicación. En las consultas periódicas de satisfacción con los socios que realiza el Club, el departamento de comunicación incluye periódicamente algunas preguntas para testar cuál es el consumo de información y qué priorizan en cuanto a contenidos. Los resultados indican que este público objetivo demanda a la institución abundante información y un alto nivel de exigencia en la

4 New Media es el resultado de la convergencia de las telecomunicaciones, los ordenadores y los medios tradicionales en el entorno digital de producción de contenidos que son distribuidos y compartidos digitalmente. Los soportes sobre los que se sustenta van desde los sitios web, blogs, correos electrónicos y redes sociales hasta la gestión de audio y video en streaming, las tecnologías para soportes móviles, la tecnología wifi o los accesos a Internet (Santomier et al., 2010: 38-39).

5 Entrevista personal mantenida en la sede del Club. 
gestión, es decir, les requieren "una constante transparencia de la gestión empresarial, deportiva y social que se lleva a cabo en el FC Barcelona”, afirma Ruiz.

Según el ex director de comunicación del Club Jordi Badía (2009), los medios se resignaron a aceptar que el Club utilizara sus propios soportes comunicativos, como FC Barcelona TV, para comunicar ciertos contenidos que preferían no exponer a través de los soportes tradicionales de la comunicación institucional como ruedas de prensa o comunicados de prensa, especialmente cuando se trataba de una estrategia en la que quedaba de manifiesto la voluntad de ser la única fuente ante socios y seguidores, sin que mediara la interpretación del periodista. No obstante, buena parte de los periodistas deportivos consideran que el hecho de que el FC Barcelona controle y gestione la información de la actualidad del Club se ha convertido en un monopolio y entienden que ese modelo de gestión comunicativa empobrece la marca y la reputación del Club (Santos, 2010).

Ante ello, desde el departamento de comunicación se insiste en su voluntad de no convertirse en un competidor con los diferentes medios y se asegura que constantemente se busca el equilibrio entre las necesidades informativas de los periodistas y los intereses corporativos de la entidad.

Para Toni Ruiz, la credibilidad del departamento de comunicación ante los periodistas se basa en que estos confíen en que no se les va a mentir. Por eso, sostiene que "es preferible no responder en los casos conflictivos para el Club, antes que dar una respuesta que pueda generar equívocos". También se ha de tener en cuenta que este comportamiento muestra la evolución del modelo comunicativo del FC Barcelona. De una época en la que la atención informativa sobre el Club no estaba masificada y en la que los periodistas departían de tú a tú con los jugadores y técnicos, estableciendo incluso relaciones personales entre ellos, se pasa al momento actual en el que los clubes blindan a los jugadores ante los medios de comunicación (Barroso, 2012).

Esta situación ha generado, según Toni Ruiz, una deshumanización de esas relaciones, ya que los jugadores no conocen a los periodistas. La ingente cantidad de medios de comunicación que siguen la actualidad del FC Barcelona tiende a aumentar las demandas sobre los protagonistas de la información.

En este contexto de presión mediática, los jugadores son los que solicitan la ayuda del departamento de comunicación para que se establezcan los filtros oportunos. La demanda de esta protección por los jugadores se produce fundamentalmente entre los deportistas que el Club ficha de otros equipos, ya que la política de comunicación del Club con los jugadores que provienen de los equipos inferiores de la entidad se basa en la educación mediática, como advierte Ruiz.

Por la propia dimensión del Club, el interés informativo de la institución no sólo se centra en la esfera deportiva. El departamento de comunicación también debe atender las peticiones de medios de otros ámbitos informativos, como la prensa económica, la internacional o la social, teniendo en cuenta, además, que la temporada deportiva realiza un parón vacacional. De ahí que desde el departamento de comunicación se implementen contenidos específicos, desde una actitud proactiva de la información, en la que la actividad que desarrolla la Fundación del FC Barcelona y sus acciones de responsabilidad social corporativa adquiere un papel determinante. En este sentido, el hecho de que la entidad tenga firmados convenios con agencias internacionales como Naciones Unidas y en la temporada 2010-2011 se incorporara 
Qatar Foundation, obligó al Club en su momento a desarrollar acciones comunicativas $^{6}$ que canalizaran la difusión mediática de esta acción social ${ }^{7}$.

Además de este comportamiento comunicativo sobre la esfera de lo social, Ruiz destaca que el departamento de comunicación del FC Barcelona desarrolla buena parte de su trabajo en colaboración con el área de Marketing, en estrategias de negocio y para posicionar la marca FC Barcelona, especialmente en los mercados futbolísticos emergentes, como Asia y Estados Unidos, que se caracterizan por el alto poder adquisitivo, interesados por el deporte y donde no existe competencia de clubes de fútbol.

Y aunque el FC Barcelona tiene un $80 \%$ de su masa social radicada en Cataluña, la estrategia empresarial está enfocada a los mercados del resto de España e internacional, dado que es el club de fútbol que mayor número de seguidores tiene en todo el mundo, con más de 340 millones de personas (Quintana, 2013).

\subsubsection{La comunicación con socios y aficionados}

EI FC Barcelona fundamenta su relación con los socios y aficionados en la proyección de la imagen e identidad del Club a través de la multiplataforma comunicativa integrada por los medios propios, que se impulsaron bajo la presidencia de Joan Laporta. En la temporada 2011-2012, además, se rediseñó la estrategia digital de la entidad (Pellicer y Ruiz, 2011) y se mejoraron las prestaciones en la mayoría de los soportes. A continuación, analizamos los cambios más significativos.

- FC Barcelona TV. Se han incorporado a la parrilla diversos espacios de tertulia sobre el funcionamiento interno del Club para que los socios conozcan los entresijos de la entidad. También emiten informativos específicos en los días de partido, programas sobre la actualidad de las diferentes secciones del Club, así como las retransmisiones en directo de partidos de las categorías inferiores de las secciones deportivas. Se puede ver, de manera gratuita, desde cualquier punto de Cataluña a través del programa temático número 2 de la Televisión Digital Terrestre (TDT) y se continúa emitiendo, a través de suscripción, en plataformas de satélite y cable, consiguiendo, en la temporada 2010-2011 una audiencia media de dos millones de espectadores ${ }^{8}$. Asimismo, las emisiones de FC Barcelona $T V$ también se pueden seguir a través de las redes sociales oficiales del Club: Twitter, Facebook y Youtube.

- Sitio web corporativo www.fcbarcelona.es. Está configurado como una plataforma comunicativa, en la que se incluyen las emisiones de R@adio FC Barcelona, la actualización de Twitter y las retrasmisiones en streaming de los diferentes eventos de FC Barcelona TV. En el mismo sitio web se incorporó en la temporada 2010-2011 la comunidad virtual de seguidores del Club (www.fcbfans.cat).

6 El presidente del Club recurrió, por ejemplo, a la rivalidad con el Real Madrid CF para trasladar al socio barcelonista las ventajas de este acuerdo, ya que los ingresos del nuevo patrocinio permitirán, según Rosell, que el FC Barcelona supere al Madrid como el club de fútbol más rico del mundo (Mundo Deportivo, 2011).

7 Con la llegada de Sandro Rossell a la presidencia del FC Barcelona, el Club incluyó el logotipo de la Qatar Foundation en la camiseta, que acompañará al de UNICEF y que supuso un importante aporte económico a la entidad (el Club ingresará más de 150 millones de euros durante las seis temporadas del acuerdo), aunque generó controversias (fundamentalmente porque contrasta que el logotipo de UNICEF tiene un carácter solidario y el de Qatar Foundation implica un reembolso económico) (Aguilar, 2010).

8 La audiencia de este soporte alcanzó picos del 3,8\%, con 169.000 espectadores, en la final de la Liga de Campeones que el FC Barcelona ganó al Manchester United en mayo de 2011. 
A finales de 2011, dentro de la estrategia digital, se incorporaron las webs de socios, peñas y empresas, con el objetivo de mantener un espacio comunicativo propio de estos stakeholders.

- Redes sociales. Integradas en el sitio web oficial, el Club se ha posicionado en Twitter, con tres cuentas en catalán, castellano e inglés, así como cuentas para las secciones de fútbol, baloncesto, balonmano, hockey sobre patines, fútbol sala y fútbol formativo. En Youtube es el canal de deportes más visto en España y en Facebook dispone de cuentas específicas para el Club, la fundación y las diferentes secciones deportivas.

- Radio B@rça. Empezó sus emisiones en 2007 y se encuentra integrada en el sitio web corporativo del Club, donde se pueden seguir todos los partidos que disputa el equipo. Junto a los periodistas deportivos de la entidad participan ex jugadores del Club como comentaristas especializados. Emite en catalán, español e inglés.

- Revista FC Barcelona. Sustituyó a la primera revista editada por el FC BarceIona, La Veu del Club. Esta publicación bimestral, con una tirada de 140.000 ejemplares, profundiza, mediante reportajes y entrevistas, en los asuntos de actualidad, al tiempo que aporta, a través de sus secciones fijas, una mirada a la historia del Club.

- Medios para los socios. Se implementan diferentes soportes dirigidos específicamente a ellos, como el boletín electrónico mensual, editado para 50.000 socios senior; el boletín electrónico mensual, dirigido a socios junior menores de 15 años; Infosocios digitales, que reciben los socios senior con noticias de última hora sobre los eventos o dónde solicitar entradas; Infojuniors, con la misma filosofía, pero dirigido a los socios junior; la revista FC Barcelona Junior, una publicación interactiva bimestral que el Club ha editado de forma que se pueda coleccionar y que incluyen contenidos de ocio para toda la familia, y el sitio web junior www.fcbjunior.cat, un espacio que complementa a las diferentes publicaciones y en el que los jóvenes seguidores barcelonistas pueden descargarse juegos y fondos de escritorio vinculados a la entidad.

De acuerdo con Pellicer y Ruiz (2011), el FC Barcelona puso en marcha a finales de 2011 una estrategia que busca consolidar el sitio web oficial del Club como una multiplataforma comunicativa, con la que generar audiencia, fidelizarla y rentabilizar los 100 millones de visitas que recibe cada temporada. Asimismo, se pretende potenciar la comunicación a través de las redes sociales para mantener el valor de marca en el ámbito internacional. Para complementar la anterior acción, el FC Barcelona llegó, en noviembre de 2011, a un acuerdo con Tencent, que ha desarrollado en China un modelo similar a Twitter y que le permite al Club acceder a un mercado de 300 millones de usuarios (Pellicer y Ruiz, 2011).

En su estrategia digital, el Club también ha apostado por los dispositivos móviles en los que desarrollar diferentes aplicaciones, tanto para smartphones, iphones y tabletas con un doble objetivo: como una vía de explotación comercial de la marca y como un instrumento para fidelizar a los seguidores vinculándolos al Club. A principios de 2012, los usuarios podían acceder a tres aplicaciones: FCB Mobile, con todo tipo de contenidos y servicios; FCB Watch, que permite consultar los lugares públicos donde se retransmiten los partidos del FC Barcelona, y Fantasy Manager, un juego interactivo donde ejercer de entrenador del equipo. 
La estrategia del FC Barcelona en los nuevos medios se basa en la creación de contenidos que les permitan atender las necesidades informativas de los socios y seguidores del Club, así como posicionarse como líderes mundiales en estos soportes, accediendo a nuevos mercados, según Rosell (Pellicer y Ruiz, 2011: 24).

\section{Conclusiones}

Los clubes de fútbol que disputan competiciones internacionales y que poseen un perfil de marca global como el FC Barcelona compiten con otras organizaciones para atraer y mantener la fidelidad de sus públicos objetivos. Los resultados deportivos de estas entidades en las diferentes competiciones en las que participan resultan relevantes, pero no lo es menos su gestión empresarial, que afecta no solo a los dirigentes y a los socios, sino también a otros grupos de interés: desde trabajadores, jugadores y técnicos hasta los seguidores del club y los medios de comunicación. Como hemos observado, la relación con sus públicos objetivos se configura, por tanto, como un área estratégica en el desarrollo empresarial de estas entidades.

En este contexto, el FC Barcelona ha evolucionado en la última década a partir de una serie de premisas que define su identidad: la profesionalización, tanto de los deportistas como de las organizaciones; la comercialización, con estrategias orientadas al marketing; el producto, que no se centra únicamente en el espectáculo deportivo, sino más bien en los ingresos que generan las acciones colaterales en torno a ese evento, y, como consecuencia de la interacción de las anteriores, los públicos participantes en la "vida" del Club: jugadores, aficionados, medios de comunicación, la ciudad, las empresas y los propietarios de la entidad.

La gestión de la comunicación de este Club se desarrolla en un ecosistema comunicativo diferente al de hace unos años, en el que participan tanto los clubes de fútbol y los jugadores como los medios de comunicación y las propias audiencias. Desde la década de los ochenta hasta finales de los noventa, la comunicación se generaba en las propias organizaciones, basada en el evento como núcleo duro de los mensajes que recogían los medios de comunicación a través de acciones de Publicity para distribuirlos a las audiencias de esos deportes, con un feedback muy limitado. En la última década, con la implantación de las TIC, el flujo informativo ha cambiado de forma radical.

Aunque se mantienen algunas de las constantes propias de la unidireccionalidad de las acciones off line, como las ruedas de prensa y los comunicados, en la actualidad la comunicación se ha convertido en un proceso bidireccional, ya que han aparecido nuevos soportes comunicativos vinculados a las TCl que permiten a las audiencias participar activamente en el flujo informativo como emisores y no sólo interactuar con sus respuestas. Al mismo tiempo, también se amplía el número de participantes en este ámbito de la comunicación deportiva, ya que los deportistas se están constituyendo en sus propios altavoces mediante el uso de las redes sociales.

Por tanto, la comunicación se ha integrado en la estructura organizacional del Club como una herramienta estratégica, que ha ido consolidándose y adquiriendo mayor protagonismo a medida que la entidad se transformaba en empresa. Asimismo, la gestión comunicativa ha mantenido su propia evolución, transitando de modelos identificados, nítidamente con los postulados clásicos de las Relaciones Públicas, a planteamientos en los que la gestión se fundamenta en dos aspectos esenciales: las 
posibilidades de interacción que permiten las TIC y la capacidad para emitir la información sobre la entidad, sin la intermediación de los medios tradicionales.

El actual modelo comunicativo de los grandes clubes parece homogeneizarse en Europa. Hace unos años, los clubes ingleses, especialmente el Manchester United, establecieron un modelo empresarial basado en la globalización de la marca a través de estrategias en las que la comunicación y el marketing se aliaban para generar contenidos comerciales. Ese comportamiento societario fue imitado por el FC Barcelona en los inicios del siglo XX y, a día de hoy, el Club continúa apostando por esta estrategia. Ahora, el comportamiento se traslada a la gestión comunicativa que los clubes realizan con los medios y los periodistas deportivos, donde el acceso a la información deportiva de los clubes está controlada por el filtro del departamento de comunicación y unificada gracias a la emisión constante de contenidos propios a través de los soportes comunicativos e informativos que crean estas entidades deportivas.

\section{Bibliografía}

Aguilar, A. (2010). Rosell tras firmar el acuerdo con Qatar Foundation: "Seguimos teniendo una camiseta solidaria". Mundo Deportivo, 18 de diciembre. Recuperado de http:// www.mundodeportivo.com/gen/20101213/54087046370/noticia/rosell-tras-firmar-elacuerdo-con-qatar-foundation-seguimos-teniendo-una-camiseta-solidaria.html.

Allen, D., y Eguía Recuero, R. (2005). FC Barcelona: cambiando las reglas del juego. Caso de estudio. Instituto de Empresa Business School. Recuperado de http://openmultimedia.ie.edu/OpenProducts/fcbarcelona/fcbarcelona/pdf/DG1_481.pdf.

Badía, J. (2009). El FC Barcelona al descobert. Badalona: Ara Llibres SCCL.

Badía, J. (2011). El FC Barcelona y la política de comunicación. El Periódico de Catalaunya, 17 de enero. Recuperado de http://www.elperiodico.com/es/noticias/barca/ barca-politica-comunicacion-667630.

Barroso, M.A. (2012). Estrellas bajo la ley del silencio. Abc, 20 de febrero. Recuperado de http://www.abc.es/20120220/deportes/abcp-estrellas-bajo-silencio-20120220.html.

Bernardos, M. (1979). Núñez prohíbe a un periodista la entrada en el Camp Nou. El País, 2 de noviembre. Recuperado de http://elpais.com/diario/1979/11/02/deportes/310345215_850215.html.

Boyle, R. y Haynes, R. (2004). Football in the New Media age. London: Routledge.

Boyle, R. y Haynes, R. (2009). Power Play. Sport, the Media and Popular Culture. Edinburgh: Edinburgh University Press Ltd.

Bravo Mayor, L.J. y Balmont, A. (2010). La sociedad de Football de Barcelona de 1894 y el "Foot-ball Club Barcelona" de 1899: ¿un mismo Club o Clubes diferentes? Cuadernos de Fútbol. Primera Revista de Historia del Fútbol Español, n 7 . Recuperado de http:// www.cihefe.es/cuadernosdefutbol/2010/02/la-sociedad-de-footbal-de-barcelona-de1894-y-el-foot-ball-Club-barcelona-de-1899-\%C2\%BFun-mismo-Club-o-Clubes-diferentes/.

Chadwick, S. y Arthur, D. (2007). Más que un Club (more than a Club): the commercial development of FC Barcelona. En S. Chadwick y D. Arthur (eds.), International cases in the business of sport (1-12). Oxford: Butterworth-Heinemann. 
Cleland, J. (2009). The Changing Organizational Structure of football Clubs and their relationship with external media. International Journal of Sport Communication, $\mathrm{n}^{\circ} 2$, 417-431.

Dávila, A., Foster, G. y Llopis, J. (2007). Futbol Club Barcelona: Globalization Opportunities. Stanford. Graduate School of Business. Case SPM-33, 1-38. Recuperado de http://miha.ef.uni-lj.si/_dokumenti3plus2/192320/view_pdf.pdf.

Escorcia, D. (2010). La comunicación del FC Barcelona. La Vanguardia, 14 de noviembre. Recuperado de http://hemeroteca-paginas. lavanguardia.com/LVE05/PUB/2010/11/14/ LVG201011140801LB.pdf.

Estanyol Casals, F. (2010). Analysis of how F.C. Barcelona implemented a virtuous circle between 2003 and 2006 to become the best football team of the world in 2009 . En 2010 International Conference on E-business, Management and Economics (ICEME2010). Hong Kong, China.

Favorito, J. (2007). Sports publicity: a practical approach. Oxford: Butterworth-Heinemann.

Fortunato, J. (2010). Pete Rozelle. Developing and communicating the sports brand. International Journal of Sport Communication, $\mathrm{n}^{\circ}$ 1, 361-377.

García Candau, J. (1979). EI FC Barcelona empieza a ser solo un Club. El País, 27 de marzo. Recuperado de http://elpais.com/diario/1979/03/27/deportes/291337212_850215. html.

García Candau, J. (1980). Núñez quiere paro. El País, 1 de octubre. Recuperado de http:// elpais.com/diario/1980/10/01/deportes/339202803_850215.html.

García, E., De Maragas, M. y Gómez, M. (1995). El deporte en las televisiones españolas: Un papel creciente en la programación. Telos, $n^{\circ} 38,110-118$.

Ginesta Portet, X. (2008). Comunicación corporativa y fútbol en la era de las TIC: los casos del FC Barcelona, Sevilla FC y RC Recreativo de Huelva. En F. Sabés y J. J. Verón (coords), Internet como sinónimo de convergencia mediática y tecnológica (153-170). Zaragoza: Asociación de la Prensa de Aragón.

Ginesta Portet, X. (2009). Les Tecnologies de la Informacio i la Comunicacio i l-esport: una anàlisi de la Primera Divisió espanyola de futbol (2006-2008). Tesis doctoral. Universitat Autònoma de Barcelona.

Ginesta Portet, X. (2010). Los medios propios de los Clubes de fútbol españoles. De la revista oficial a los canales de TDT. Estudios sobre el mensaje periodístico, $\mathrm{n}^{\circ} 16$, 145-166.

Ginesta, X. y Casino, R. (2008). Corporate Communication in a Global Sports Media Complex: The Case Study of FC Barcelona. En R. Oglesby y M. G. Adams (eds.), Business Research Yearbook: Global Business Perspectivas (389-395). Beltsville, Maryland: International Academy of Business Disciplines.

Ginesta, X., Gómez, M., y Serrat, J. (2011). Observatorio FC Barcelona 2010. La campaña a la presidencia del FC Barcelona en la prensa deportiva y las redes sociales. Ámbitos, $\mathrm{n}^{\circ}$ 20, 197-215.

Glaser, B. (1992). Basics of Grounded Theory Analysis. Mill Valley, California: Sociology Press. 
Gómez, S., Martí, C., y Opazo, M. (2010). Value creation from the organizational structure of a sports entity. En S. Gómez, K. Kase e I. Urrutia (eds), Value creation and sport Management (176-208). Cambridge: Cambridge University Press.

Hamil, S., Walters, G. y Watson L. (2010). The Model of Governance at FC Barcelona: Balancing Member Democracy, Commercial Strategy, Corporate Social Responsibility and Sporting Performance. Soccer \& Society, 11 (4), 474-504. DOI: http://dx.doi. org/10.1080/14660971003780446.

Hernández, A. (1976). Crisis en el Club de Montal. El País, 3 de noviembre. Recuperado de http://elpais.com/diario/1976/11/03/deportes/215823604_850215.html.

Hutchins, B. y Rowe, D. (2010). Reconfiguring Media Sport for the Online World: An Inquiry Into Sports News and Digital Media. International Journal of Communication, $\mathrm{n}^{\circ}$ 4, 696-718.

Jones, D. (1994). Medios de comunicación deportivos: La situación española en el contexto internacional. Telos: Cuadernos de Comunicación, Tecnología y Sociedad, $\mathrm{n}^{\circ} 38$, junio-agosto, 101-108.

Jones, D. (2004). La globalización comunicativa en Cataluña: procesos y tendencias. ZER, Revista de Estudios de Comunicación, 16 (9), 27-43.

López, T. (1999). Un siglo de mutua dependencia. La Vanguardia, 5 de noviembre. Recuperado de http://hemeroteca.lavanguardia.com/preview/1998/11/01/pagina-69/34148720/pdf.html?search=collegi.

Miserachs, J. y Polo, E. (1999). La tele del campeón. Mundo Deportivo, 27 de mayo. Recuperado de http://hemeroteca-paginas.mundodeportivo.com./EMD03/HEM/1999/05/27/ MD19990527-020.pdf.

Moragas, M., Kennett, C y Ginesta, X. (2011). Football and media in Europe. A new sport paradigma for the global era. En A. Tomlinson, C. Young y R. Holt (eds.), Sport and the Transformation of Modern Europe. States, media and markets 1950-2010 (128-149). Oxon (Reino Unido): Routledge.

Mundo Deportivo (1997). El FC Barcelona presenta su web. 12 de junio. Recuperado de http://hemeroteca-paginas.mundodeportivo.com./EMD03/HEM/1997/06/12/ MD19970612-017.pdf.

Mundo Deportivo (2000). Carta abierta de Televisió de Catalunya a Josep Lluis Núñez. Recuperado de http://hemeroteca-paginas.mundodeportivo.com./EMD02/ PUB/2000/04/01/EMD20000401018MDP.pdf.

Mundo Deportivo (2001). Toni Ruiz, nuevo jefe de prensa del Club. 23 de agosto. Recuperado de http://hemeroteca-paginas.mundodeportivo.com./EMD03/PUB/2001/08/23/ EMD20010823015MDV.pdf.

Mundo Deportivo (2011). El FC Barcelona ya tiene a tiro al Real Madrid en cuanto a ingresos. 16 de septiembre. Recuperado de http://hemeroteca-paginas.mundodeportivo. com./EMD03/PUB/2011/11/16/EMD20111116005MDP.pdf.

Ordeix, E. y Ginesta, X. (2009). Aplicación de los parámetros de la excelencia en la gestión de relaciones con los medios de comunicación: una comparativa entre FC Barcelona y RCD Espanyol. En M. C. Carretón Ballester (coord.), La gestión de las relaciones con los públicos (102-118). Alicante: Asociación de Investigadores en Relaciones Públicas (AIRP). 
Pedersen, P. M., Miloch, K. S. y Laucella, P. C. (2007). Strategic Sport Communication. Champaign, IL: Human Kinetics.

Pellicer, M., y Ruiz, M. (2011). La estrategia digital del líder. FC Barcelona. Revista oficial del FC Barcelona, $\mathrm{n}^{\circ}$ 54, 23-26. Recuperado de http://media3.fcbarcelona.com/media/asset_publics/resources/000/009/188/original/FCB54_Desembre_CAST_baixa. v1325669623.pdf.

Perearnau, F. (2003). Un ejemplo de buena gestión. Mundo Deportivo, 10 agosto. Recuperado de http://hemeroteca-paginas.mundodeportivo.com./EMD03/PUB/2003/08/10/ EMD20030810018MDP.pdf.

Portabella, J. (2010). Barcelona. Identitat y cosmopolitisme. Paradigmes, $n^{\circ} 5$. Recuperado de http://paradigmes.cat/numero-5/us-de-la-marca-de-pais-al-mon-i-a-catalunya/ barcelona-identitat-i-cosmopolitanisme/.

Quintana, T. (2013). El FC Barcelona tiene 340 millones de culés por el mundo. Sport, Barcelona. Recuperado de http://www.sport.es/es/noticias/planeta-barca/ barca-tiene-340-millones-cules-por-mundo-2761720.

Relaño, A. (2012). Nacidos para incordiarse. Un siglo de agravios entre el Madrid y el FC Barcelona. Madrid: Ediciones Martínez Roca.

Rodríguez de Paz, A. (1999). Arranca el Canal FC Barcelona. La Vanguardia, 28 de julio. Recuperado de http://hemeroteca-paginas.lavanguardia.com/LVE01/PUB/1999/07/28/ BAR19990728-011.pdf.

Sanahuja Peris, G. (2013). Planificación y estrategia de identidad corporativa en los Clubes deportivos españoles. Questiones publicitarias, v. I, n 18, 126-143.

Sanchís, A. (1999). Una relación de 100 años. Mundo Deportivo, 5 de noviembre. Recuperado de http://hemeroteca-paginas.mundodeportivo.com./EMD03/HEM/1999/11/05/ MD19991105-014.pdf.

Santomier, J., y Costabiei, A. (2010). New Media Challenges in the Twenty-First Century. En S. Hamil y S. Chadwick (eds.), Managing Football: An International Perspective (37-54). Oxford: Butteworth-Heinemann.

Santos Fernández, A. (2010). L'Entorn. El circ mediàtic del FC Barcelona. Valls: Cossetània Edicions.

Schultz, B., Caskey, P. y Esherick, C. (2010). Media Relations in Sport. West Virginia: Fitness Information Technology.

Segura, M. (2004). Novedades en el área de comunicación. Mundo Deportivo, 11 de noviembre. Recuperado de http://hemeroteca-paginas.mundodeportivo.com./EMD02/ PUB/2004/11/11/EMD20041111014MDP.pdf.

Serras, M. (2008). Ricard Maxenchs, pionero de las relaciones públicas en el fútbol. El País, 22 de octubre. Recuperado de http://elpais.com/diario/2008/10/22/necrologicas/1224626401_850215.html.

Xifra, J. (2008). Soccer, civil religion, and public relations: Devotional-promotional communication and Barcelona Football Club. Public Relations Review, 34 (2), 192-198. DOI: http://dx.doi.org/10.1016/j.pubrev.2008.03.005. 
